numerically of the order of Iooo in that region, but $\Delta p / p$ cannot be neglected in calculations requiring greater accuracy, or in the upper reaches of the atmosphere, where $p$ has a much smaller value because $\theta$ does not fall proportionally to the fractional fall of $p$.

In considering any physical explanation of the structure of the atmosphere, the difference $\Delta p / p-\Delta \theta / \theta$ is an important quantity. In fact, as a rule, it appears that, somewhere or other in a vertical section of the troposphere (where $\Delta \theta$ and $\Delta p$ are of the same sign), in consequence of the variations in the magnitudes involved, the quantity $\Delta p / p-\Delta \theta / \theta$ becomes zero and changes sign. To that curious circumstance is due the dominance of the influence of the stratosphere upon the dynamics of the surface layers, although it only represents about a quarter of the whole mass of the atmosphere. In the stratosphere $\Delta p$ and $\Delta \theta$ are of opposite signs, and their influences in the production of pressure difference reinforce each other. Hence in the stratosphere, pressure differences are rapidly built up, while in the troposphere changes are capricious and contradictory.

But fortunately these considerations are, so far as can be judged, of little importance in the cases to which $\mathrm{Mr}$. Cave has applied his rough and ready formula, and do not affect the general accuracy of his conclusions.

For the practical study of the dynamics of the atmosphere we are largely dependent upon observations with pilot balloons. They may be taken as supplementing observations of clouds, and, in due time, both must be brought into relation with the observations of pressure and temperature obtained from registering balloons. It is in many ways unfortunate that the track of a registering balloon cannot always be followed by a theodolite or otherwise determined. As it is, we often get our kinematical conditions from one occasion, and our baric and thermic conditions from a different one.

Something may be done to bring the two together by means of observations of cloudsequence, which can be observed on either occasion. At present these have hardly come within the range of meteorological work. Few observers are effectively conscious of the rapidity of the changes which are indicated by clouds, and which must be the results of the distribution of pressure, temperature, and wind.

In the book before us little is said of the association of cloud-forms and cloud-changes with the variations of the structure of the atmosphere disclosed by pilot balloons, but that part of the subject has great possibilities, and this leads us to express the hope that in a subsequent edition of this interesting work Mr. Cave may be able to give us the benefit of his experience in that direction also.

W. N. Shaw.

\section{SOCIOLOGY AND MEDICINE.}

(I) The Task of Social Hygiene. By Havelock Ellis. Pp. $x v+414$. (London: Constable and Co., Ltd. IgI 2.) Price $8 s .6 d$. net.

(2) The People's Medical Guide: Points for the Patient, Notes for the Nurse, Matter for the Medical Adviser, Succour for the Sufferer, Precepts for the Public. By Dr. John Grimshaw. Pp. $x x+839$. (London: J. \& A. Churchill. 1912.) Price $8 s$. 6d. net.

(x) THE title of this book somewhat masks I the nature of its contents, for by "social hygiene" the author means to convey the study of those things which concern the welfare of human beings living in societies. The various chapters, or essays as they practically are, include such varied subjects as the changing status of woman and the woman's movement, eugenics and love, religion and the child, the falling birth-rate, sexual hygiene, war against war, international language, and others. The author generally presents the two points of view, supporting them by quotations and summaries from many sources. The essays are interesting reading, but at the end leave us somewhat in doubt as to what would be for the best, or what the writer considers would be best.

(2) This book covers almost the whole range of subjects comprised within the scope of the practice of medicine and surgery, including the specialities such as diseases of the throat and eye. The information given seems generally to be accurate, is imparted in simple language, and important points are frequently driven home by some terse sentence, e.g. "a tooth in the head is worth two on the plate" (p. 57). Some capital sections are given on the management of children, diets and cooking, and physical exercises. The matter does not always seem to come quite in the right place, and simple domestic remedies and treatment may be omitted; for example, that common complaint of children, "child crowing," or "spasmodic croup," is scarcely noticed under children's ailments, but is relegated to the chapter on diseases of the throat, and it is certainly by nc means "invariably" associated with rickets.

We think that the compass of the work is somewhat beyond that necessary or desirable for the general public, but the volume would serve as an excellent book of reference for the district nurse, health visitor, missionary, ship's captain, and the like.

R. T. H.

NO. 2200 , VOL. 927 